\title{
Fast reoptimization of Steiner trees: Changing the Edge set
}

\author{
Subhash Panwar \\ Motilal Nehru National institute of technology- \\ Allahabad
}

\author{
Suneeta Agarwal \\ Motilal Nehru National institute of technology- \\ Allahabad
}

\begin{abstract}
In this paper, we discuss the problem of reoptimization of Steiner tree. We are given an instance of Graph and also an optimal Steiner tree of it. If some changes occurs later on in the given graph. New optimal Steiner tree is to be determine, this process is known as re optimization. We consider two cases of change: one is addition of a new edge and second is, Deletion of an existing edge. For both the cases, we provide approximation algorithms with corresponding approximation ratio equal to $(1+\delta)$ where $0<\delta<1$.
\end{abstract}

\section{Keywords:}

Steiner tree, approximation algorithm reoptimization.

\section{INTRODUCTION}

The Steiner tree problem is one of the most famous combinatorial optimization problems in network design. In traditional optimization theory, we consider the task to find an optimal or close to optimal solution to the given instance of optimization problem. In many applications it is necessary to re-compute the solution after some modification has

So it leads to theory of reoptimization problems. Why one does requires to re compute the solution for a little modification? Learn from previous optimal solutions and try to get good solution quickly.

Steiner tree problem is well known to be NP-hard. Many approximation algorithms are given to compute the Steiner tree for a given instance of graph [1].

The algorithm to find minimum Steiner tree, by simple approximation method [1]: Compute the shortest paths between all pairs of terminal vertices and then compute a minimum-cost spanning tree over the shortest-path weighted complete graph with vertex-set R. Removal of redundant edges might be needed in order to transform the tree so computed to a Steiner tree of G. The approximation ratio achieved by this algorithm is bounded above by 2.

The problem we deal in this paper is reoptimization of Steiner minimal tree problem. We are given an optimal solution for given graph. And we wish to maintain a good solution efficiently, when the instance gets slightly modified. The modification may be adding a node, adding an edge and removing a node and removing an edge. The adding and removing node have been addressed in [3]. Here we address the case of adding new edge and removing existing edge. We propose the simple procedures for tackling the case. These procedures are mainly based on the shortest path and minimum spanning tree computation. And we also find the goodness of the solution by approximation ratio.

Two reoptimization variants of the Steiner tree problem where the local modifications consist of adding one vertex to the graph and deleting one vertex from the graph, respectively were considered in [2]. In this paper we deal with addition and deletion of an edge from the graph. We propose an algorithm for each case.

Our main results are as follow:

1. We design approximation algorithms for both addition and deletion of a single edge.

2. We show that for in both cases of reoptimization the approximation ratio is $(1+\delta)$. Where $0<\delta<1$. And which depends on the number of adjacent edges of e.

The paper is organized as follows. In Section 2, we formally define the problem and give some definitions. In section 3 and 4 , we present approximation algorithm for adding a new edge and deleting an edge respectively with mathematical calculation of approximation ratio. Section 5 concludes the paper.

\section{PRELIMINARIES}

We are given a weighted graph $\mathrm{G}(\mathrm{V}, \mathrm{E}, \mathrm{C})$ where $\mathrm{V}$ is set of vertices and $E$ is set of edges, and a weight function $C, C: E \rightarrow R^{+}$. And we are also given a set $\mathrm{R} \subseteq \mathrm{V}$, which denotes a set of terminals. We also assume here that the weight function $\mathrm{C}$ satisfies the triangle inequality $(\mathrm{c}((\mathrm{u}, \mathrm{v})) \leq \mathrm{c}((\mathrm{u}, \mathrm{w}))+\mathrm{c}((\mathrm{w}, \mathrm{v}))$ for all $u, v, w \in V)$. We call such a graph a metric graph.

The degree of a vertex is denoted as $\operatorname{deg} G(u)$ for $u \in V$. The length of path is its number of edges. In the shortest path, the length of the path is minimized whereas in a cheapest path its cost is minimized. For two sub graphs of G: $\mathrm{T} 1, \mathrm{~T} 2 \subseteq \mathrm{G}$, we denote CheapestEdge (T1, T2) as the cheapest edge in G connecting T1 and $\mathrm{T} 2$ directly.

\section{ADDITION OF AN EDGE}

Let $\mathrm{G}$ be a metric graph, $\mathrm{R}$ be a terminal set. We assume that an optimal solution $T_{0}$ of the given metric graph $G$ is known. Now if a new edge e $(u, v)$ is introduced to the graph. The Modified graph is denoted as $G_{m}$. An Approximate Steiner tree $T_{A}$ is to be obtained for $\mathrm{G}_{\mathrm{m}}$. For this we propose an algorithm EdgeAdd. 


\section{Algorithm EdgeAdd:}

1. Add new edge $\mathrm{e}(\mathrm{u}, \mathrm{v})$ in $\mathrm{T}_{0}$.

2. Find the Cycle formed in $\mathrm{T}_{0}$. and delete an edge having maximum weight from cycle formed in $\left\{\mathrm{T}_{0} \mathrm{U}\right.$ e $\}$ and Let the new tree be $\mathrm{T}_{\mathrm{A}}$.

3. Compare $\mathrm{T}_{\mathrm{A}}$ and $\mathrm{T}_{0}$

4. If still $\mathrm{T}_{0}$ is having smallest cost than solution is equal to $T_{0}$ and is an exact solution of graph $G_{m}$.

5. Else $T_{A}$ is approximate solution of graph $G_{m}$.

Theorem 1: Algorithm EdgeAdd achieves an approximation ratio of $(1+\boldsymbol{\delta})$, where $0<\delta<1$.

\section{Case 1:}

Let $T_{m}$ be optimal Steiner tree after modification. If the new edge is not a part of $T_{m}$, than $T_{0}$ will the exact solution of $G_{m}$.

\section{Case 2:}

Here the case, where e $(\mathrm{u}, \mathrm{v}) \in \mathrm{E}\left(\mathrm{T}_{\mathrm{m}}\right)$ is considered.

Let $\mathrm{f}_{1} \ldots \ldots \ldots \ldots \mathrm{f}_{\mathrm{k}} \in \mathrm{E}\left(\mathrm{T}_{\mathrm{m})}\right.$ be the edges adjacent to $\mathrm{e}$ in $\mathrm{T}_{\mathrm{m}}$. Let $\mathrm{fl}=\left(\mathrm{u}, \mathrm{x}_{1}\right)$ and let $\mathrm{g}_{1}=\left(\mathrm{x}_{1}, \mathrm{v}\right)$

Then the feasible solution for $(G, S)$ is $T_{m}-e+g_{1}$

Means,

$\mathrm{C}\left(\mathrm{T}_{0}\right) \leq \mathrm{C}\left(\mathrm{T}_{\mathrm{m}}\right)-\mathrm{C}(\mathrm{e})+\mathrm{C}\left(\mathrm{g}_{1}\right)$

By triangular inequality: $\quad \mathrm{C}\left(\mathrm{g}_{1}\right) \leq \mathrm{C}\left(\mathrm{f}_{1}\right)+\mathrm{C}(\mathrm{e})$

So we can say for each edge $f_{i}$ adjacent to e in $T_{m}$ that it holds:

$$
\mathrm{C}\left(\mathrm{T}_{0}\right) \leq \mathrm{C}\left(\mathrm{T}_{\mathrm{m}}\right)+\mathrm{C}\left(\mathrm{f}_{\mathrm{i}}\right)
$$

And

$\mathrm{C}\left(\mathrm{T}_{\mathrm{A}}\right)=\mathrm{C}\left(\mathrm{T}_{0}\right)+\mathrm{C}(\mathrm{e})-\mathrm{C}\left(\mathrm{f}_{\max }\right)$

Where $f_{\max }=$ Most expensive edge on the path from $u$ to $\mathrm{v}$ in $\mathrm{T}_{0}$. And $\mathrm{C}\left(\mathrm{f}_{\max }\right) \geq \mathrm{C}(\mathrm{e})$.

If we say that a particular edge $\left(f_{i}\right)$ is a part of the $T_{m}$ than we can say that

$\mathrm{C}\left(\mathrm{f}_{\mathrm{i}}\right) \leq \delta \mathrm{C}\left(\mathrm{T}_{\mathrm{m}}\right) \quad$ Where $0<\delta<1$

Hence,

$$
\begin{aligned}
\mathrm{C}\left(\mathrm{T}_{\mathrm{A}}\right) & \leq \mathrm{C}\left(\mathrm{T}_{\mathrm{m}}\right)+\mathrm{C}\left(\mathrm{f}_{\mathrm{i}}\right)+\mathrm{C}(\mathrm{e})-\mathrm{C}\left(\mathrm{f}_{\max }\right) ; \\
& \leq \mathrm{C}\left(\mathrm{T}_{\mathrm{m}}\right)+\delta \mathrm{C}\left(\mathrm{T}_{\mathrm{m}}\right)+\mathrm{C}\left(\mathrm{f}_{\max }\right)-\mathrm{C}\left(\mathrm{f}_{\max }\right) \\
& \leq \mathrm{C}\left(\mathrm{T}_{\mathrm{m}}\right)+\delta \mathrm{C}\left(\mathrm{T}_{\mathrm{m}}\right) \\
& \leq(1+\delta) \mathrm{C}\left(\mathrm{T}_{\mathrm{m}}\right)
\end{aligned}
$$

\section{DELETION OF AN EDGE}

Let $\mathrm{G}$ is a metric graph, $\mathrm{S}$ is terminal set and we assume that the optimal solution of the metric graph $\mathrm{G}$ is known and denotes as $\mathrm{T}_{0}$ and now an edge e $(\mathrm{u}, \mathrm{v})$ that is part of $\mathrm{T}_{0}$ come for deletion. For this we propose given algorithm EdgeRemove.

\section{Algorithm EdgeRemove:}

1. Remove edge e $(u, v)$ from $T_{0}$.

2. Find the cheapest path $P$ from $u$ to $v$ on $G$

3. $\mathrm{T}^{\prime}=\mathrm{T}_{0} \mathrm{U} \mathrm{P}\{$ Add $\mathrm{P}$ to $\mathrm{Ts}\}$

4. Find the Minimum spanning tree $\mathrm{T}_{\mathrm{A}}$ of $\mathrm{T}^{\prime}$

5. Return $\mathrm{T}_{\mathrm{A}}$.

Theorem 2: Algorithm EdgeRemove achieves an approximation ratio of $(1+\boldsymbol{\delta})$, where $0<\delta<1$.

Case 1:

Let $T_{m}$ be optimal Steiner tree after modification. If the edge e is not a part of $T_{0}$, than $T_{0}$ will the exact solution of $G_{m}$.

Case 2:

We consider the case when e $\in \mathrm{E}\left(\mathrm{T}_{0}\right)$ and $\mathrm{e} \notin \mathrm{E}\left(\mathrm{T}_{\mathrm{m}}\right)$ and for it there will $\mathrm{C}\left(\mathrm{T}_{0}\right) \leq \mathrm{C}\left(\mathrm{T}_{\mathrm{m}}\right)$.

Let $f_{1} \ldots \ldots \ldots . . . . . f_{k}$ be the edges adjacent to in $T_{0}$.

Let assume an edge $f_{i}=(u, w) \in E\left(T_{0}\right)$, which is also part of $T_{m}$. than

$\mathrm{C}\left(\mathrm{f}_{\mathrm{i}}\right) \leq \delta \mathrm{C}\left(\mathrm{T}_{\mathrm{m}}\right)$.

Let $\mathrm{g}(\mathrm{w}, \mathrm{v})$,

Than, $\mathrm{C}\left(\mathrm{T}_{\mathrm{A}}\right) \leq \mathrm{C}\left(\mathrm{T}_{0}\right)-\mathrm{C}(\mathrm{e})+\mathrm{C}(\mathrm{g})$

By triangular equality: $\mathrm{C}(\mathrm{g}) \leq \mathrm{C}(\mathrm{e})+\mathrm{C}\left(\mathrm{f}_{\mathrm{i}}\right)$

Hence,

$$
\begin{aligned}
\mathrm{C}\left(\mathrm{T}_{\mathrm{A}}\right) \leq \mathrm{C}\left(\mathrm{T}_{0}\right)+\mathrm{C}\left(\mathrm{f}_{\mathrm{i}}\right) \\
\leq \mathrm{C}\left(\mathrm{T}_{\mathrm{m}}\right)+\delta \mathrm{C}\left(\mathrm{T}_{\mathrm{m}}\right) \\
\leq(1+\delta) \mathrm{C}\left(\mathrm{T}_{\mathrm{m}}\right)
\end{aligned}
$$

\section{CONCLUSION}

We have presented a fast reoptimization algorithm for Steiner tree problem. We have handled insertion of a new edge in initial graph and also deletion of an edge from graph. We have provided reoptimization techniques which achieve $(1+\delta)$ approximation, where $0<\delta<1$.

\section{REFERENCES}

[1] Bang Ye Wu and Kun-Mao Chao, Spanning tree and optimization problem, in Chapman \& Hall/CRC Press,USA,2004.

[2] F. Hwang, D.Richards, P.Winter The Steiner tree problem, in Annals of Discrete Mathematics,Vol.53 North-Holland,1992.

[3] B. Escoffier, M. Milanic, V.T. Paschos, Simple and fast reoptimizations of Steiner tree problem. DIMACS Techn. Report 2007-01, 2007.

[4] S. E. Dreyfus and R. A. Wagner. The Steiner problem in graphs. Networks, 1:195-207, 1972.

[5] Bil`o, D., B“ockenhauer, H.-J., Hromkovi`c, J., Kr'alovi`c, R., M"omke, T., Widmayer, P., Zych, A.: Reoptimization of Steiner trees. In: Gudmundsson, J. (ed.) SWAT 2008. LNCS, vol. 5124, pp. 258-269. Springer, Heidelberg (2008)

[6] Cs0821 Sa120976 
[7] B“ockenhauer, H.-J., Hromkovi č, J., Kr'alovi`c, R., M“omke, T., Rossmanith, P.: Reoptimization of Steiner trees: Changing the terminal set. Theoretical Computer Science.
[8] D.B. West, Introduction to Graph Theory, 2nd edn, Prentice Hall Inc., Upper Saddle River, NJ, 2000.

[9] Pr"omel, H.J., Steger, A.: The Steiner Tree Problem. Advanced Lectures in Mathematics. Friedr. Vieweg \& Sohn, Braunschweig (2002) 